\title{
Endoscopic ultrasound-guided fine-needle aspiration for diagnosis of an infectious mediastinal mass and/or lymphadenopathy:
}

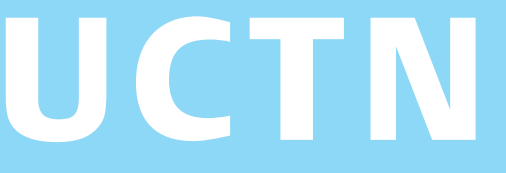

\section{Three patients with masses caused by infection with Nocardia species, Mycobacterium tuberculosis, and Mycobacterium kansasii}

Endoscopic ultrasound (EUS) can be useful in the diagnosis of infection, providing material for both cytomorphologic diagnosis and for fungal/mycobacterial cultures. We present here three cases where EUS provided a diagnosis of mediastinal infection.

The first patient was a 60-year-old man who presented with a productive cough and was found to have a $3.5-\mathrm{cm}$ mediastinal mass on computed tomography (CT). Fine-needle aspiration (FNA) of the mass was performed under EUS guidance (EUS-FNA) (Figure 1): the aspirate consisted of fibrinopurulent exudate and branching bacilli (Figure 2 ). These findings were suggestive of a Nocardia infection and this was confirmed by cultures. The second patient was a 26-year-old HIV-positive man who presented with a nonproductive cough. A chest CT scan showed mediastinal lymphadenopathy, and radial EUS showed a large subcarinal lymph node mass (Figure 3). EUS-FNA of the subcarinal lymph nodes was performed, and the aspirates revealed caseating necrosis and many acid-fast bacilli (Figure 4). Cultures of an EUS-derived sample later grew Mycobacterium tuberculosis. The third patient was a 35-yearold HIV-positive man who presented with worsening cough despite completing a course of antibiotic treatment for methicillin-resistant Staphylococcal aureus (MRSA) and $M$. kansasii. Chest CT scan revealed a mediastinal mass. EUSFNA of the mass was performed to rule out neoplasia (Figure 5), and Kinyoun staining of the aspirate was positive for long, coarsely beaded, acid-fast bacilli

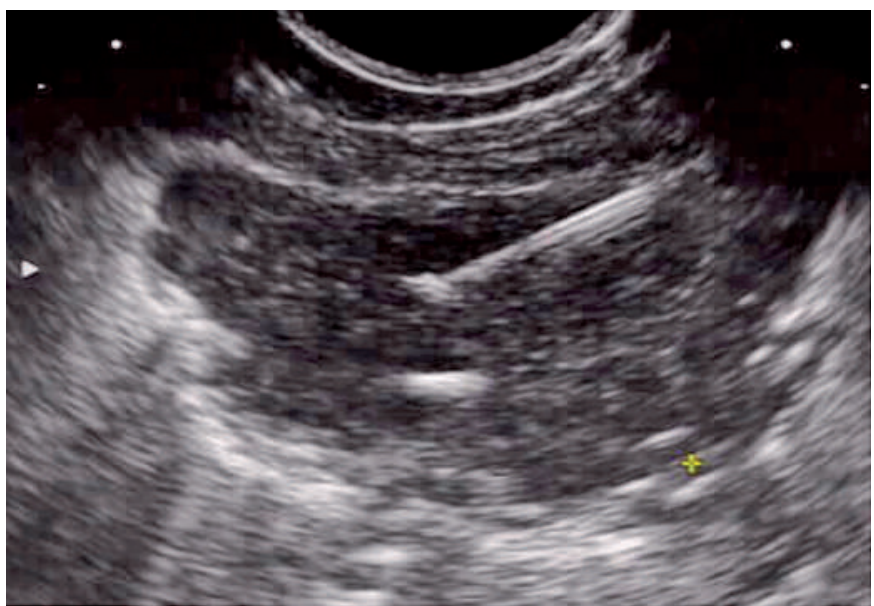

Figure 1 Endoscopic ultrasoundguided fine-needle aspiration (EUSFNA) of a mediastinal mass later revealed to be caused by a Nocardia infection.

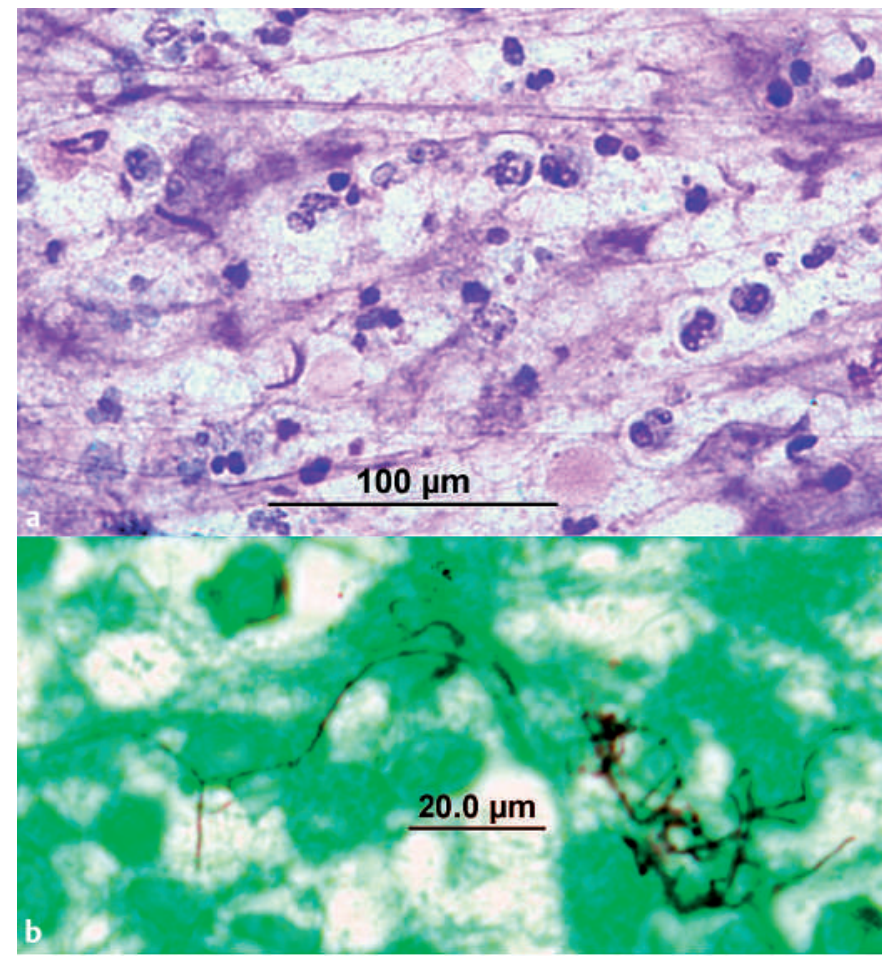

Figure 2 Histological views of the aspirate obtained by EUS-FNA from the patient in Figure 1, who had a mediastinal mass caused by a Nocardia infection. a Inflammatory exudate composed of fibrin and degenerating neutrophils was seen after staining with a Romanowsky stain (Quik-Dip).

b Branching, beaded bacilli, approximately $1 \mu \mathrm{m}$ in width, were seen on the Gomori methenamine silverstained slide. 


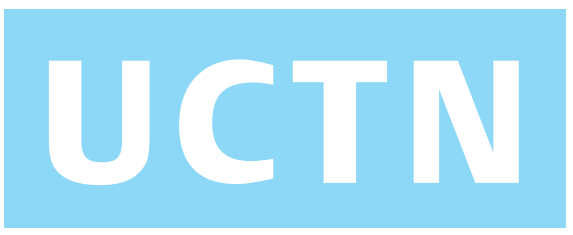

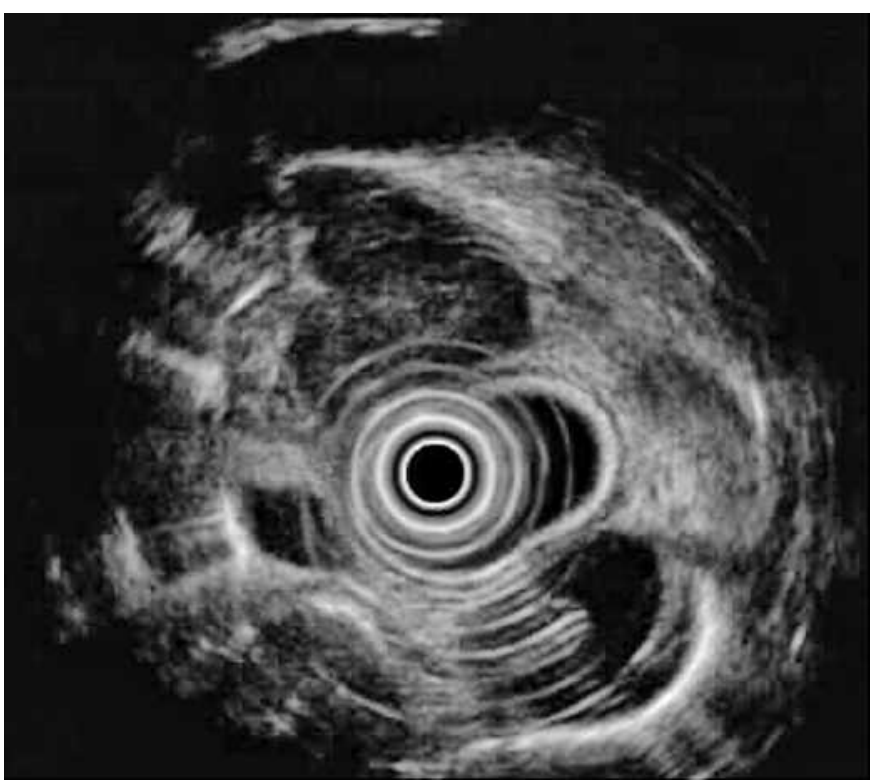

E100

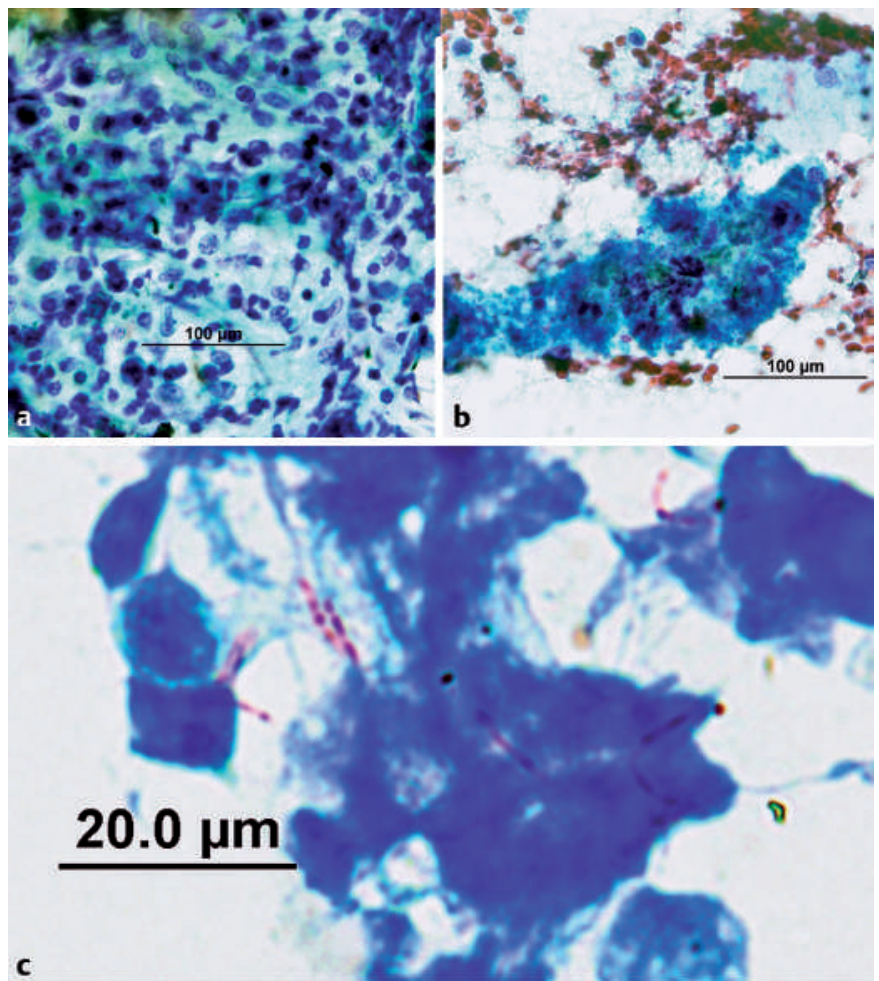

Figure 3 Radial EUS view of a large subcarinal lymph node mass in the second patient.

Figure 4 The EUSFNA aspirate from the patient in Figure $\mathbf{3}$ showed mycobacteriosis in the mediastinal lymph node. a Aggregates of macrophages, suggestive of granuloma formation. b Granular amorphous material, typical of caseous necrosis (Papanicolaou stain). c Short, beaded, acid-fast bacilli seen after staining with Kinyoun acid-fast stain. with curved ends, the typical appearance of $M$. kansasii (Figure 6).

There are a few isolated reports of infections diagnosed by EUS. We present a series of three cases of mediastinal lesions diagnosed by EUS. In all three, the preliminary diagnosis of an infectious condition was made by EUS, and in two out of the three the organism was cultured from EUS-derived material. EUS-FNA diagnosis of infection in the mediastinum allowed for prompt diagnosis and treatment of the infection.

There have been a few isolated reports of tuberculosis diagnosed by EUS-guided FNA [1-5], but to our knowledge there are no reported cases of Nocardia or $M$. kansasii infections being diagnosed this way. As the use of EUS-FNA becomes more widespread we are likely to see more cases and reports of infectious diagnoses made by EUS.

Endoscopy_UCTN_Code_CCL_1AF_2AC

\section{T. Chaya ${ }^{1}$, V. Schnadig ${ }^{3}$, P. Gupta ${ }^{2}$, \\ R. Logrono ${ }^{3}$, M. S. Bhutani ${ }^{1}$}

${ }^{1}$ Center for Endoscopic Ultrasound, Division of Gastroenterology, University of Texas Medical Branch, Galveston, Texas, USA

2 Department of Medicine, University of Texas Medical Branch, Galveston, Texas, USA 

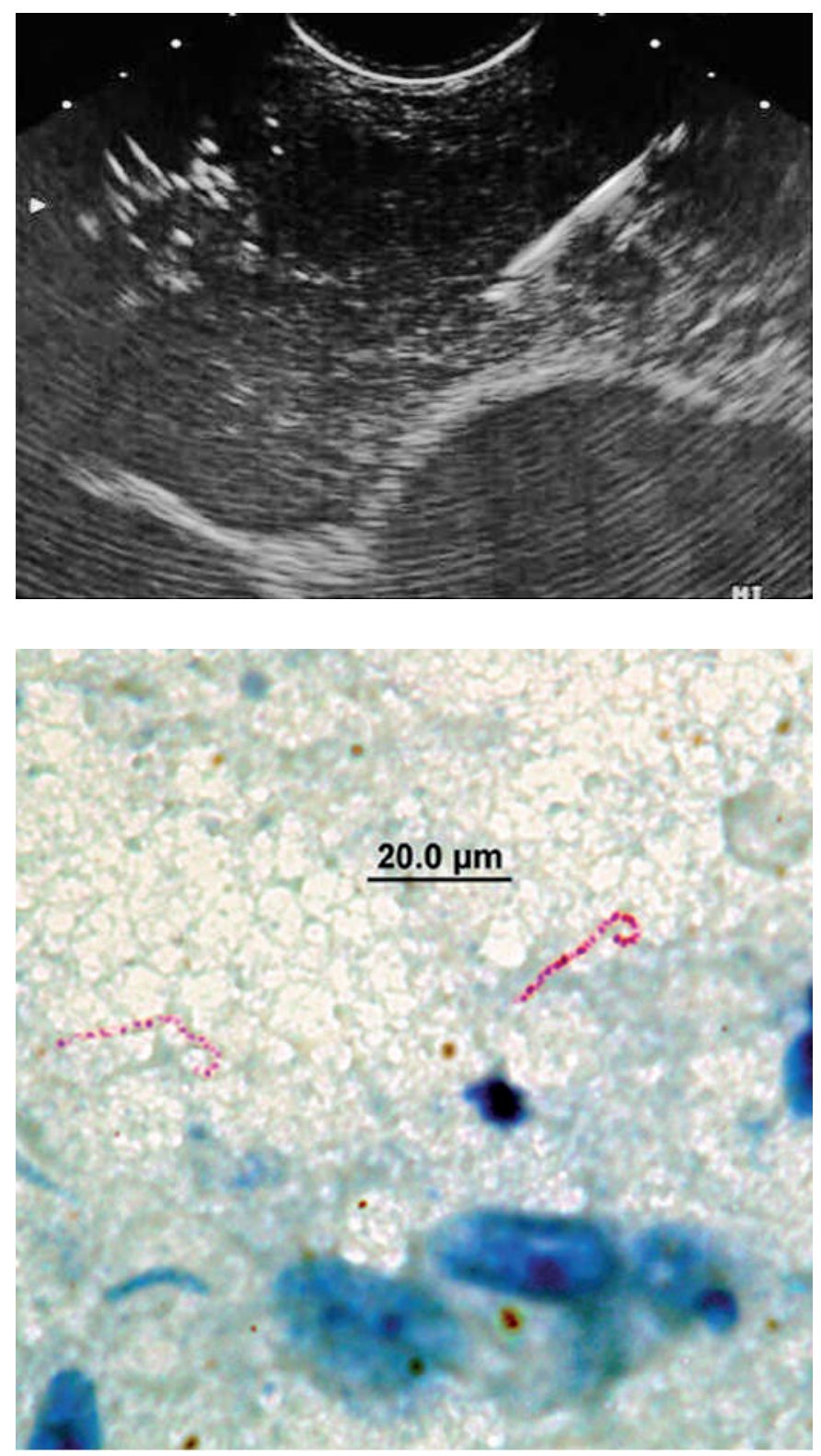

Figure 5 Linear EUS-FNA of a mediastinal mass in patient 3, who was found to have a Mycobacterium kansasii infection.

Figure 6 Mycobacterium kansasii in the mediastinal mass aspirated by EUSFNA (patient 3). These were very long, coarsely beaded, acid-fast bacilli that commonly had curved ends (Kinyoun acid-fast stain).

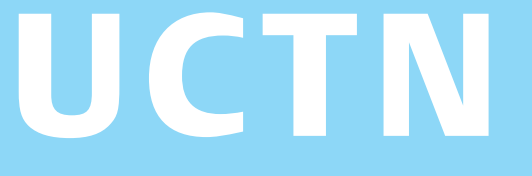

${ }^{3}$ Department of Cytopathology, University of Texas Medical Branch, Galveston, Texas, USA.

\section{References}

${ }^{1}$ Kitmacher P, Bourhis F, Zarski JP et al. A rare cause of upper digestive tract hemorrhage: an esophageal fistula in mediastinal tuberculous adenopathy. Ann Gastroenterol Hepatol (Paris) 1991; 27: 173-176

${ }^{2}$ Fritscher-Ravens A, Sriram P, Topalidis T et al. Diagnosing sarcoidosis using endosonography-guided fine-needle aspiration. Chest 2000; 118: 928 -935

${ }^{3}$ Kramer H, Nieuwenhuis JA, Groen HJ, Wempe JB. Pulmonary tuberculosis diagnosed by esophageal endoscopic ultrasound with fine-needle aspiration. Int J Tuberc Lung Dis 2004; 8: 272-273

${ }^{4}$ Khoo KL, Ho KY, Nilsson B, Lim TK. EUS-guided FNA immediately after unrevealing transbronchial needle aspiration in the evaluation of mediastinal lymphadenopathy: a prospective study. Gastrointest Endosc 2006; 63: 215-220

${ }^{5}$ Walsh PR, Williams DB. Mediastinal adenopathy: finding the answer with endoscopic ultrasound-guided fine-needle aspiration biopsy. Intern Med J 2005; 35: 392 - 398

\section{Corresponding Author}

\section{S. Bhutani, M.D.}

Center for Endoscopic Ultrasound University of Texas Medical Branch 301 University Blvd.

Route 0764

Galveston

Texas 77555-0764

USA

Fax: $\quad+1-409-772-4789$

E-mail: msbhutan@utmb.edu 